\title{
Monte Carlo study of a highly efficient gas ionization detector for megavoltage imaging and image-guided radiotherapy
}

\author{
H. Keller, ${ }^{\text {a) }}$ M. Glass, and R. Hinderer \\ Department of Medical Physics, University of Wisconsin, Madison, Wisconsin 53706-1532 \\ K. Ruchala \\ Department of Medical Physics, University of Wisconsin, Madison, Wisconsin 53706-1532 \\ and Tomotherapy, Inc., Middleton, Wisconsin 53706 \\ R. Jeraj \\ Department of Medical Physics, University of Wisconsin, Madison, Wisconsin 53706-1532 \\ G. Olivera and T. Rock Mackie \\ Department of Medical Physics, University of Wisconsin, Madison, Wisconsin 53706-1532 \\ and Tomotherapy, Inc., Middleton, Wisconsin 53706
}

(Received 19 March 2001; accepted for publication 21 November 2001; published 25 January 2002)

\begin{abstract}
The imaging characteristics of an arc-shaped xenon gas ionization chamber for the purpose of megavoltage CT imaging were investigated. The detector consists of several hundred $320 \mu \mathrm{m}$ thick gas cavities separated by thin tungsten plates of the same thickness. Dose response, efficiency and resolution parameters were calculated using Monte Carlo simulations. The calculations were compared to measurements taken in a $4 \mathrm{MV}$ photon beam, assuming that the measured signal in the chambers corresponds to the therein absorbed dose. The measured response profiles for narrow and broad incident photon beams could be well reproduced with the Monte Carlo calculations. They show, that the quantum efficiency is $29.2 \%$ and the detective quantum efficiency at zero frequency $\mathrm{DQE}(0)$ is $20.4 \%$ for the detector arc placed in focus with the photon source. For a detector placed out of focus, these numbers even increase. The efficiency of this kind of radiation detector for megavoltage radiation therefore surpasses the reported efficiency of existing detector technologies. The resolution of the detector is quantified with calculated and measured line spread functions. The corresponding modulation transfer functions were determined for different thicknesses of the tungsten plates. They show that the resolution is only slightly dependent on the plate thickness but is predominantly determined by the cell size of the detector. The optimal plate thickness is determined by a tradeoff between quantum efficiency, total signal generation and resolution. Thicker plates are more efficient but the total signal and the resolution decrease with plate thickness. In conclusion, a gas ionization chamber of the described type is a highly efficient megavoltage radiation detector, allowing to obtain CT images with very little dose for a sufficient image quality for anatomy verification. This kind of detector might serve as a model for a future generation of highly efficient radiation detectors. (C) 2002 American Association of Physicists in Medicine.
\end{abstract}

[DOI: $10.1118 / 1.1445414]$

Key words: megavoltage photon radiation detector, megavoltage CT, quantum efficiency, resolution

\section{INTRODUCTION}

In radiotherapy, a radiation dose as high as necessary is given to the tumor while applying a dose as low as possible to specified tissues near the target volume. Not only it is necessary to guarantee safety and accuracy of the delivery procedure, with multifraction treatments it is also necessary to guarantee reproducibility on a daily basis. New application and optimization techniques allow the delivery of dose distributions which highly conform to the shape of the tumor and/or conformally avoid sensitive structures. However, daily setup variations, tumor shape changes and organ motion contribute to geometrical uncertainties in the position of the target and the avoidance volumes. For these reasons, sev- eral early studies have suggested that patient positioning be checked daily. ${ }^{1}$ Consequently, much effort has been devoted to the development of new methods to image the patient just prior or even during the treatment. Current portal imaging techniques allow only a projection of the treatment volume on a plane perpendicular to the beam axis behind the patient.

The ultimate form of verification imaging, however, is "image-guided radiotherapy," which intends to provide three-dimensional images of the entire patient's anatomy of interest. To this purpose, much current research effort has been devoted to the optimization of detector technologies combined with the modification of current delivery systems.

Concerning the modification of delivery systems, different approaches to image-guided radiotherapy are under develop- 


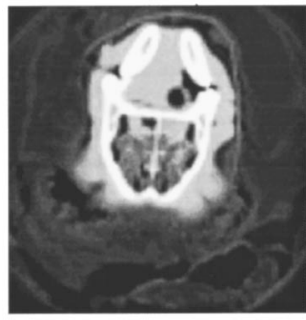

$\mathrm{kVCT}$

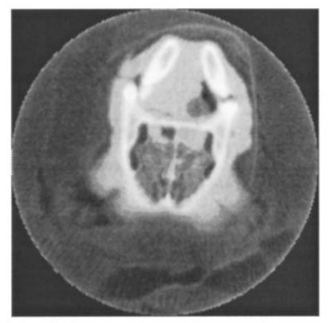

MVCT
FIG. 1. Comparison of a kVCT (left) and an MVCT image (right) of a dog cadaver head. The head was packed within an expandable foam package. The kVCT image was taken on a Siemens HiQ CT scanner and the MVCT image was acquired on the Tomotherapy Benchtop. The dose used for the MVCT image was 2 cGy.

ment, which can be categorized in two groups: (i) megavoltage CT (MVCT) imaging, and (ii) on-board kilovoltage CT $(\mathrm{kVCT})$ imaging possibly combined with megavoltage portal imaging or MVCT. Using the open (uncollimated) and/or the shaped treatment beam from a linac has been proposed for many years as a method of verifying the position of the patient during treatment (portal imaging). Such megavoltage portal images have become clinically common in the last couple of years. However, MVCT imaging is being investigated as a three-dimensional (3D) alternative. Many systems have been proposed for multislice cone beam CT using purpose-built detectors $^{2-4}$ or using commercial EPIDs such as fluoroscopic systems, ${ }^{5-7}$ liquid-filled ionization chamber ${ }^{8}$ or an amorphous silicon flat-panel detector. ${ }^{9}$ The first tomotherapy prototype utilises an arc-shaped one-dimensional detector array for fan-beam megavoltage CT. ${ }^{10-12}$

Two approaches combining the treatment unit with an onboard kilovoltage CT system are under development. The first uses a medical linear accelerator equipped with an additional $\mathrm{kV}$ x-ray source to produce high-contrast $\mathrm{kV}$ radiographs along with the existing megavoltage portal imaging capabilities. ${ }^{13}$ In the tomotherapy approach treatment and imaging capabilities are combined by rotating a kilovoltage and a megavoltage fan-beam around the patient. Benchtop experiments showed, that megavoltage images of a canine cadaver head with comparable high-contrast resolution as a kVCT scan can be obtained using only a few cGy dose. ${ }^{14}$ This was achieved by using a conventional arc-shaped CT xenon gas ionization detector. Even clinically more relevant doses of 2 cGy per scan have been documented (see Fig. 1). In the MVCT image, sufficient low-contrast details are visible. As with on-board kVCT, MVCT can be used to reconstruct images during the treatment itself. ${ }^{14}$

On-board kVCT will generally provide superior images to MVCT, however, for the purpose of anatomy verification, diagnostic image quality may not be needed. Moreover, we consider MVCT to be superior to EPIDs. In addition, we feel that the capabilities of megavoltage CT imaging are not yet fully explored. The challenge for megavoltage imaging lies in the fact, that such systems usually suffer from lower subject contrast at megavoltage energies and the fact, that highenergetic photons transfer much larger energies in matter re- quiring that fewer be used. ${ }^{15}$ In addition, the efficiency of MV photon detection with detectors as described above, with the exception of the first detector, is much lower than for typical $\mathrm{kV}$ detectors. In order to improve the low-contrast detectability, a high signal-to-noise ratio (SNR) of the detector signal is needed which can be achieved by keeping the noise as low as possible and/or by increasing the number of signal generating photons (quantum efficiency of the detector). The mentioned detector uses a $2 \mathrm{D}$ array of scintillation crystal detector elements made of $\mathrm{CsI}(\mathrm{Tl})$, thallium-doped cesium iodide, instead of the phosphor screen of conventional metal/phosphor screen devices. ${ }^{2-4}$ The thickness of the scintillation crystals was chosen to yield a relatively high quantum efficiency of $18 \%$, however, further increasing the thickness of the crystals has its limitations. ${ }^{3}$ The work of this paper is intended to make some steps towards an optimized detector for megavoltage CT imaging using a different concept of improving efficiency.

Image-guided radiotherapy intends to close the loop of planning, delivery, and verification. The possibility to obtain an image of the patient during each fraction and to calculate on it the dose delivered leads automatically to the concept of "adaptive radiotherapy." 16 Based on verification images, an intra- or interfractional feedback mechanism can be employed in order to modify the delivery between fractions or even within the same fraction, in order to fulfill the original prescription. ${ }^{17}$ However, the basis for image-guided radiotherapy and therefore for adaptive radiotherapy is a fast, efficient and reliable image detector.

The University of Wisconsin Tomotherapy Benchtop uses a conventional arc-shaped CT xenon gas ionization detector. In this work, some physical and imaging characteristics of this detector were studied by means of Monte Carlo calculations. In particular, parameters characterizing detection efficiency (detective quantum efficiency at zero frequency, quantum detection efficiency) and spatial resolution (modulation transfer function) were characterized. For the current design of the detector, the results were compared to measurements. In view of a future detector design, the efficiency and resolution properties were also studied for different geometrical parameters of the detector, in particular for different septal plate dimensions.

\section{MATERIALS AND METHODS}

All the measurements were done on the University of Wisconsin's (UW) Tomotherapy Benchtop. The Tomotherapy Benchtop is a table-top setup of the proposed tomotherapy gantry. ${ }^{11,18}$ It consists of an ORION linear accelerator (General Electric Medical Systems, Buc, France) with a nominal acceleration potential of $4 \mathrm{MV}$, a rotary table with a linear stage to translate the phantom vertically, and an arcshaped xenon detector. The linear accelerator is removed from its original gantry and is permanently mounted parallel to the horizon. The isocenter of the Benchtop is defined to be in the center of the table at $93 \mathrm{~cm}$ distance from the source.

In order to perform Monte Carlo simulations of the radiation response of a detector, a model with three essential com- 
ponents is needed: (i) a model of the photon source, (ii) an accurate geometrical description of the detector and the materials involved, and (iii) a model for the signal formation and signal read-out in the detector. The following subsections describe these components.

\section{A. Photon source}

The geometry of the head of the ORION accelerator was modelled in BEAM and a phase space file of the emerging particles was scored at a distance of $18.7 \mathrm{~cm}$ downstream from the target. This phase space description was used for the direct comparison of measured and calculated fan beam responses.

For the calculation of the detector line spread functions a point source has to be used. Therefore, the line spread functions were calculated with a simplified photon source, i.e., an isotropic point source with a 4 MV spatially uniform energy spectrum. It was assumed, that for the study of efficiency and resolution of the detector, the results will not be greatly influenced by the exact description of the photon source using the complete phase space description. The energy spectrum used was a spatially averaged energy spectrum over a small radial field area around the central axis extracted from the phase space file. For the comparison of the calculated and measured small beam responses, the point source was replaced by a finite sized circular disk source with Gaussian intensity distribution and the same $4 \mathrm{MV}$ energy spectrum.

\section{B. The arc detector}

The detector in use at the UW Tomotherapy Benchtop is an arc-shaped CT xenon detector (General Electric). As the original drawings were not available, the geometrical parameters were estimated based upon extensive measurements. The detector consists of 738 detector cells each comprising of two gas cavities divided by a thin tungsten septal plate. The distance between the two plates defines the size of a single gas cavity. The separation is $0.32 \mathrm{~mm}$, which is also the thickness of the plates. The septal plates are $2.54 \mathrm{~cm}$ long (in the beam direction). At every odd plate a high-voltage of $+300 \mathrm{~V}$ is applied. The even plates act as charge collecting electrodes for the charge produced in the gas cavities, which are filled with xenon gas under high pressure for which 5 atm was used in the Monte Carlo calculations. The charge produced in two adjacent xenon cavities is collected together to yield the signal of an detector cell (channel). Consequently, a cell has a size of $1.28 \mathrm{~mm}$. The extension of the detector in lateral beam direction is $5.4 \mathrm{~cm}$. On top (upstream) and at the bottom of the detector, a front and a back plate made of aluminum of $0.1 \mathrm{~cm}$ and $0.5 \mathrm{~cm}$ thickness respectively, are mounted. For the energy under consideration (4 MV) as well as for the energy of the prototype gantry $(6 \mathrm{MV})$, the front plate acts as a filter for contaminant electrons.

The front face of the detector is placed $129.2 \mathrm{~cm}$ away from the photon source. As the radius of the detector arc measures only $103.6 \mathrm{~cm}$, the geometrical center of the detector is slightly off-focus with the source. The detector focus

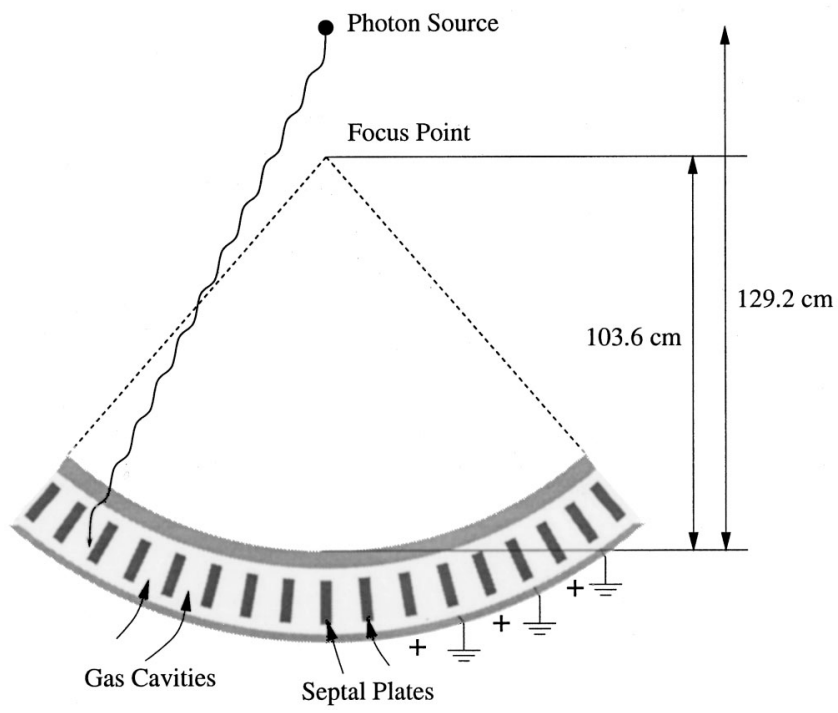

FIG. 2. Schematic of the simulation geometry. The gas cavities and septal plates are enclosed in an aluminum case, the front and back plates of which are indicated as a gray-shaded area. The geometrical center of the detector (focus point) is $25.6 \mathrm{~cm}$ beneath the photon source. The polarities of the septal plates are indicated in the right half of the detector.

point is $25.6 \mathrm{~cm}$ away from the photon source. The geometry is depicted in Fig. 2.

The detector geometry is implemented as a component module (ARCCHM) within the EGS4/BEAM Monte Carlo code. ${ }^{19}$ For each part of the component module different dimensions and materials can be specified. The absorbed dose was scored in separate dose scoring regions for the xenon cavities and the tungsten plates.

The BEAM code in the version BEAM99 from the National Research Council of Canada (NRC) served as the computational tool for the particle transport through the detector geometry. In general, the particle cutoff parameters ECUT and PCUT were chosen as low as possible, i.e., 0.521 $\mathrm{MeV}$ and $0.01 \mathrm{MeV}$, respectively. The parameter ESTEPE was set to 0 so that the PRESTA boundary crossing algorithm was invoked. In all the calculations, no variance reduction techniques were used. The simulations were performed on an $633 \mathrm{MHz}$ AlphaPC 164LX under Linux.

For the calculation it was assumed, that the signal read out from each channel is proportional to the total absorbed energy or the absorbed dose in the xenon cavities. This is justified, since a detector channel acts as a simple ionization chamber, as shown in Fig. 2. The absorbed dose of two neighboring xenon cavities was averaged to yield a measure for the signal in a single channel. The absorbed dose, thus obtained, was compared to the measured signal.

\section{Detector response profiles}

For the broad beam response profile, $10^{8}$ photon histories were sampled from the ORION phase space file. 750 xenon cavities and 749 tungsten plates were defined in the ARCCHM module, a total of 1499 dose scoring regions.

In order to characterize the radiation transport within the detector and to understand the shape of the response profiles, 
different simulations were performed to obtain a particle-byparticle tracking. For these tracking calculations, the simulated field length was $30 \mathrm{~cm}$ at the isocenter (at $93 \mathrm{~cm}$ ). The width of the field was $3.89 \mathrm{~cm}$ at the isocenter in order to cover the entire detector area in the perpendicular direction. For each of the 750 xenon cavities and the 749 tungsten plates, the number and energy of photons and electrons entering and leaving were scored. The photons were further differentiated into "primary photons" not having interacted before and "scattered photons," which already had at least one interaction in the detector. The corresponding modifications were done in the BEAM code.

\section{Efficiency}

In order to characterize the imaging performance of a detector, the transfer of the signal-to-noise ratio per resolution element has to be investigated. This is described by the $d e-$ tective quantum efficiency $D Q E$ at each spatial frequency $f$. It has been demonstrated, that measurements of the frequencydependent $\mathrm{DQE}$ can be reproduced to a reasonable degree of accuracy by theoretical calculations based on a cascaded systems formalism. ${ }^{20}$ However, in this work, we only concentrate on the first two stages of the cascade. The first stage is a gain stage and describes the quantum (detection) efficiency $Q E$, or the probability of detecting a single incident photon (quantum). Its variance is described by absorbed energy distributions (AED). The second stage is the (spatial) spreading stage, as expressed in the modulation transfer function (MTF) and is covered in the next subsection.

The absorbed energy distribution AED $(E, E i)$ describes the probability that one incident photon of energy $E_{i}$ will deposit an amount of energy between $E$ and $E+d E$ in the sensitive volume of the detector. ${ }^{21}$ This distribution was obtained with Monte Carlo calculations. From these distributions, the DQE at zero frequency $\mathrm{DQE}(0)$ can be calculated. For a single incident energy $E_{i}$, it can be shown, that $\mathrm{DQE}(0)$ can be obtained from the first and second moments $M_{1}$ and $M_{2}$ of the AED distribution, ${ }^{22}$

$$
\operatorname{DQE}(0)_{E_{i}}=\frac{S N R_{\mathrm{out}}^{2}}{S N R_{\mathrm{in}}^{2}}=\frac{S N R_{\mathrm{out}}^{2}}{\frac{\left(N E_{i}\right)^{2}}{N E_{i}^{2}}}=\frac{1}{N} \frac{M_{1}\left(E_{i}\right)^{2}}{M_{2}\left(E_{i}\right)},
$$

where

$$
M_{k}\left(E_{i}\right)=\sum_{j} E_{j}^{k} \operatorname{AED}\left(E_{j}, E_{i}\right) .
$$

The summation goes over all bins $j$ holding the absorbed energies. For a polyenergetic incident photon beam with a (non-normalized) fluence spectrum $\Sigma N_{i}\left(E_{i}\right), \mathrm{DQE}(0)$ was directly calculated with the non-normalized AED distributions $N \operatorname{AED}\left(E_{j}, E_{i}\right)$, which is equivalent to a weighted version of equation (1),

$$
\operatorname{DQE}(0)_{\mathrm{poly}}=\frac{\frac{\left[\Sigma_{j} E_{j} N \operatorname{AED}\left(E_{j}, E_{i}\right)\right]^{2}}{\sum_{j} E_{j}^{2} N \operatorname{AED}\left(E_{j}, E_{i}\right)}}{\frac{\left[\sum_{i} N_{i} E_{i}\right]^{2}}{\sum_{i} N_{i} E_{i}^{2}}}=\frac{\frac{\left[\Sigma_{i} N_{i} M_{1}\left(E_{i}\right)\right]^{2}}{\sum_{i} N_{i} M_{2}\left(E_{i}\right)}}{\frac{\left[\sum_{i} N_{i} E_{i}\right]^{2}}{\sum_{i} N_{i} E_{i}^{2}}} .
$$

$N=\Sigma N_{i}$ is the total number of photons used to produce the absorbed energy distributions. Note, that the zeroth moment $M_{0}$ of the non-normalized AED simply gives the area under the AED, which is the total number of detected photons. As a photon either interacts or does not interact, $M_{0} / N$ describes the fraction of detected incident photons which is called the quantum efficiency of the detector structure.

The quantum efficiency in form of the detection probability was also investigated for a single tungsten plate separated from the array structure of the detector. This was done in order to study the influence of the neighboring tungsten plates on the efficiency of a single cell. In particular, the detection probability in a single tungsten plate $p_{\text {det }}$ was studied as a function of its thickness. $p_{\operatorname{det}}$ was defined as

$$
p_{\text {det }}=\frac{N_{\gamma}^{\text {det }}}{N_{\gamma}}=\frac{N_{c}^{\text {out }, 0}}{N_{\gamma}}
$$

where $N_{\gamma}^{\text {det }}$ is the number of detected photons and $N_{\gamma}$ is the number of photons incident on the tungsten plate. The number of detected photons was determined by calculating $N_{c}^{\text {out }, 0}$, the number of charged particles (electrons and positrons) leaving the septa plate (or "liberated") in lateral direction. The " 0 " indicates, that only the first charged particle produced from each photon is counted. To calculate this ratio, EGS4/BEAM was modified to study the particle transport through a rectangular parallelepiped of variable $X, Y$, and $Z$ dimensions. A rectangular photon beam of the same cross-section as the plate was directed perpendicular to one of its surfaces. In each simulation, the photons were directed along the $Z$-axis towards the $X-Y$ surface, where the $X$-dimension of the plate varied between 50 and $600 \mu \mathrm{m}$. The dimensions in $Y$ and $Z$ were kept constant at $5.4 \mathrm{~cm}$ and $2.54 \mathrm{~cm}$, respectively, the same dimensions as the plates in the arc detector. The ratio of the lateral area of a single half cell $(640 \mu \mathrm{m} \times 5.4 \mathrm{~cm})$ and the lateral area taken by the tungsten plate can be regarded as a fill factor. The particle fluence exiting the plate in lateral direction was scored in a phase-space file. In addition, the first charged particle produced by a single photon and leaving the plate was flagged and counted separately. Finally, as the detection probability was calculated for constant incident photon fluence, they were also rescaled in order to represent probabilities per particle hitting the plate.

In order to investigate the implications of the efficiency of the first gain stage on the measured signal, the total absorbed energy in all the xenon gas cavities was calculated for incident thin slit beams. As total absorbed energy is a measure for the total detector signal, this calculation allows some conclusions to be drawn about the expected signal-to-noise ratio. 


\section{E. Resolution}

The second stage of a detector system is described by the spatial spreading of the "signal carriers" (in our case electrons and positrons) within the detector material. The spatial spreading is uniquely characterized by investigating the response to an incident elementary point or slit beam in which case the response is called a point or line spread function (PSF and LSF), respectively.

The concept of the point spread function (PSF) and its associated modulation transfer function (MTF) is usually based on linear and shift-invariant imaging systems. Although the array detector discussed in this work is a linear system, it is clearly not shift-invariant. An incident pencil beam hitting a tungsten plate will cause a very different response than a pencil beam hitting a xenon cavity. As an additional consequence and unlike in homogeneous detector structures, the signal can not be recorded at arbitrary spatial locations but only at the positions of the gas cavities. Despite these peculiarities, a presampled point spread function was obtained in the following way: Incident pencil beams were simulated hitting a parallel flat array structure of $320 \mu \mathrm{m}$ thick tungsten plates and xenon gas cavities. This mimicks the situation for a center cell of the arc detector, where the plates point back towards the photon source. Monte Carlo calculations were done with variable plate thicknesses of 50 , $100,320,400$, and $500 \mu \mathrm{m}$ for a fixed detector cell size of $1.28 \mathrm{~mm}$. The photon energy spectrum of the pencil beams was the 4 MV ORION spectrum described above. The incident position of the pencil beams was varied in increments of $1 / 20$ of the cell size, which was $0.064 \mathrm{~cm}$, therefore achieving an oversampling factor of 20 . The number of simulated photon histories was $2 \times 10^{6}$ for beams hitting a tungsten plate and $2 \times 10^{8}$ for beams hitting a gas cavity to guarantee similar statistics. The PSF was then constructed in a "backward" approach: the absorbed energy from each pencil beam after correcting for the different incident particle histories was recorded only in the two central xenon cavities, and the corresponding signal value was then assigned to the spatial location of the incident pencil beam.

To measure the response function of an incident slit beam, thin slits of various width were produced by two pieces of 10 $\mathrm{cm}$ thick tungsten plates with carefully machined surfaces. Metal spacer ("shim") pieces were taped between the plates to produce a diverging slit with focus point at the photon source. The shim pieces had a tolerance of $0.02 \mathrm{~mm}$, which allowed the opening width of the slit to be defined very accurately. The jaws of the linac were set such that they collimated the photon field down to a size of $1 \times 1 \mathrm{~cm}^{2}$. In addition to the tungsten pieces and the jaws, two lead bricks of $4.8 \mathrm{~cm}$ thickness were placed upstream towards the photon source to reduce head scatter radiation through the slit even further. The tungsten pieces and lead bricks were placed on a table between accelerator head and detector, such that the entrance side of the slit was at the isocenter $(93 \mathrm{~cm}$ from the source) and the exit side pointed towards the center of the detector. In order to align the slit, a diode chamber was attached at the exit side of the slit. The table was rotated until

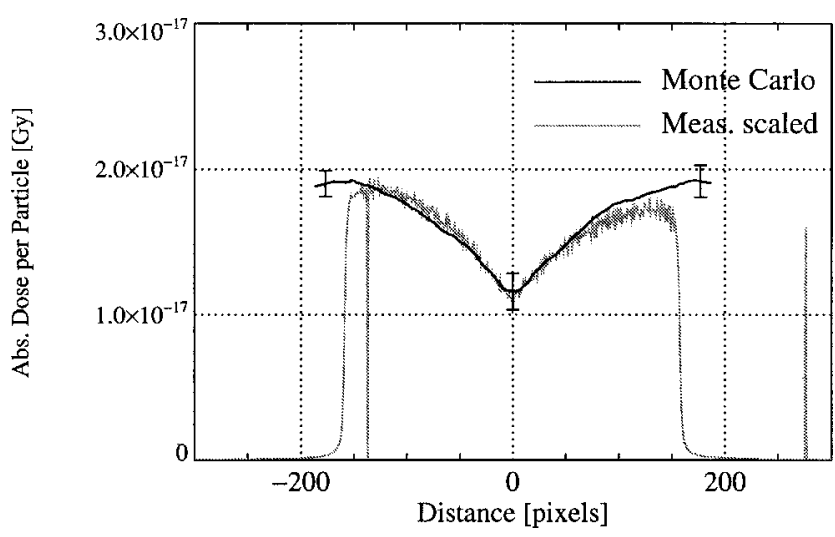

FIG. 3. Broad beam response profile to $10^{8}$ histories from the ORION phase space source. The black line is the absorbed dose per source particle in the detector channels. The curve was smoothed with a Savitzky-Golay filter. The error bars indicate the statistical error of the Monte Carlo calculation. The measured signal (light gray) was scaled to match the value of the calculation in the center. The fluctuations in the measurement from channel to channel is due to variations in the volume of the ion chambers. One channel at the position -143 gave a zero response.

the signal in the diode chamber reached its maximum. This procedure allowed for a precise alignment of the slit towards the photon source.

In this particular experiment, the slit width of the beam was defined such that exactly one detector cell was covered with radiation. In order to produce such a slit, shim pieces of $0.84 \mathrm{~mm}$ and $0.94 \mathrm{~mm}$ had to be used at the entrance and the exit side of the slit, respectively. After aligning with the diode chamber, different slit beam responses were measured by translating the table in steps of $100 \mu \mathrm{m}$. The alignment of the slit after translation was repeatedly checked by attaching the diode. This procedure was done in order to define exactly the relative position of the beam center to the collecting electrode.

The measurements were compared to Monte Carlo calculated small beam responses. The slit was modelled with the JAWS component module. The center of the slit pointed directly towards a collecting electrode. The source was a finite sized circular disk of $10 \mathrm{~mm}$ diameter. The photon fluence across the disk was a Gaussian intensity distribution with a standard deviation of $\sigma=1 \mathrm{~mm}$, the known focal spot size of the ORION accelerator.

\section{RESULTS}

\section{A. Detector response profiles}

Figure 3 shows the calculated and measured response of the detector to the photons from the ORION phase space source. Although the incident photon fluence intensity exiting the head of the accelerator is only slightly nonuniform along the fan beam, the dose per particle in xenon exhibits a sharp increase with increasing distance from the center of the detector. The measured profile shown in Fig. 3 is the average of 1000 acquired profiles. Therefore, the fluctuations in the measured profile are due to time-independent structural noise alone. The measured response profile is well reproduced by 


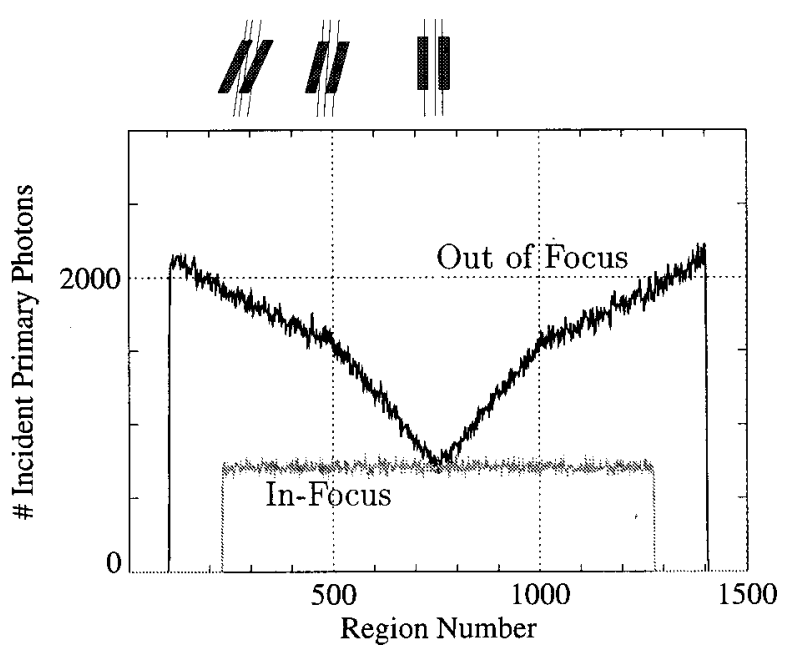

FIG. 4. Number of primary photons incident on the tungsten plates for $10^{6}$ source photon histories. On the abscissa the number of the scoring region is indicated. A total number of 1499 scoring regions was defined. The black line shows the results for the detector placed out of focus with the photon source as indicated in Fig. 2. The insets on the top show the direction of the photon beam relative to the orientation of the tungsten plates at different locations on the detector. As a comparison, the number of primary photons for the detector placed in focus with the photon source is shown as a gray line.

the Monte Carlo calculations. The calculated profile is a little wider than the measured one, because the uncollimated phase space was used for the calculation. The slight differences between the measurement and the simulation in the right part of the field are due to an asymmetry in the measured profiles. These asymmetries are due to slight misalignment of the detector such that the detector focus is not on the beam axis between the photon source and the center of the front face of the detector (see Fig. 2). Several tests showed that misalignment of the detector was a highly sensitive source of distortion of the profile shape.

In order to further investigate the form of the profiles, the number of primary photons incident on each of the tungsten plates is shown in Fig. 4 and the incident energies flux on each of the tungsten plates in Fig. 5. These "particle tracking" calculations were done for $10^{6}$ photon histories. For the off-focus position, the number of primary photons increased with increasing distance from the center, whereas for the in-focus case, as expected, this number is essentially constant across the beam (Fig. 4). The increase of the primary photons is approximately linear, which is explained by the (approximately) linear increase in cross-sectional area with increasing distance from the central axis for small angles. From Fig. 4 it is seen, that for distances larger than about 250 regions in each direction, the increase in the number of primaries changes slightly its slope. It can be shown that this corresponds to the point at the detector, where a ray emerging from the photon source is beginning to intersect two tungsten plates. Consequently, the attenuation is higher resulting in a decreased slope. This behavior is also reflected in the incident energy flux (Fig. 5).

However, for both positions of the detector, the majority of the photons striking the tungsten plates are scattered pho-

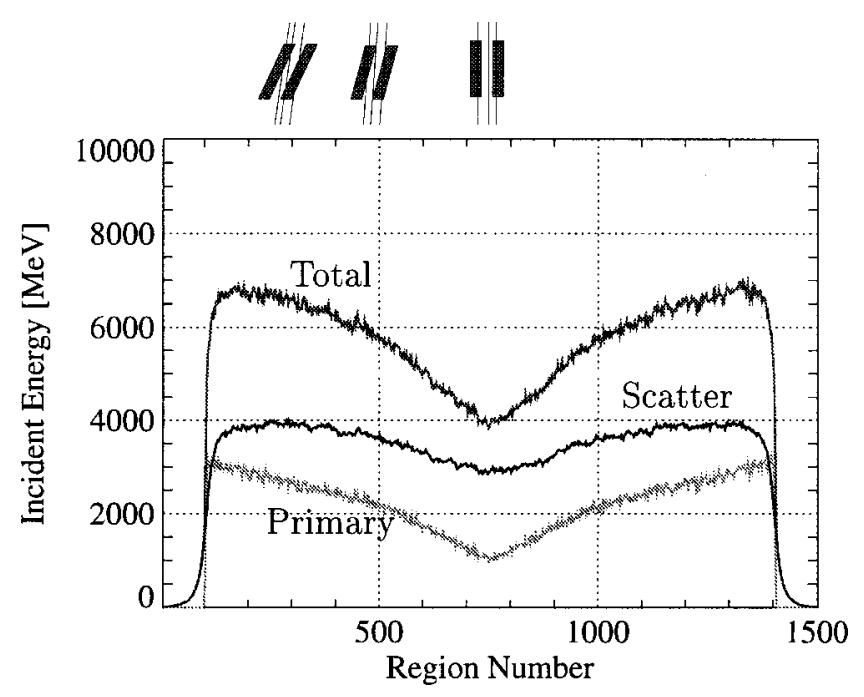

FIG. 5. Primary, scattered and total incident photon energy (flux) on the tungsten plates for $10^{6}$ source photon histories. On the abscissa the number of the scoring region is indicated. A total number of 1499 scoring regions was defined. The detector is placed out of focus with the photon source as indicated in Fig. 2. The insets on the top show the direction of the photon beam relative to the orientation of the tungsten plates at different locations on the detector.

tons which also carry the major part of the incident energy (see Table I). Although the incident energy from scattered photons is larger than the incident energy from primary photons, it is essentially constant across the detector. Therefore, the shape of the response profiles reflect primarily the distribution of the number and incident energy of the primary photons across the detector and is therefore a consequence of the detector geometry with respect to the photon source. In fact, in the off-focus position, the cross sectional area of the tungsten plates is larger due to the slight angle between the incident photons and the longitudinal axis of the septa plates. A summary of the results for $10^{6}$ incident photon histories is given in Table I.

\section{B. Efficiency}

Figure 6 shows the detection probability in the single plate $p_{\text {det }}$ as a function of the plate thickness. It is clearly seen, that the detection probability per photon hitting the plate increases with decreasing plate thickness. For example, the detection probability for the $0.32 \mathrm{~mm}$ thick plates is 0.18 and for the $0.05 \mathrm{~mm}$ thick plate $p_{\text {det }}$ is 0.59 , which means

TABLE I. Total number of photons and total energy incident on all the tungsten plates for the in-focus position for $10^{6}$ source photons.

\begin{tabular}{lcclcc}
\hline \hline & \multicolumn{2}{c}{ Number $\left[10^{6}\right]$} & & \multicolumn{2}{c}{ Energy $\left[10^{6} \mathrm{MeV}\right]$} \\
\cline { 2 - 3 } \cline { 5 - 6 } & In focus & Off focus & & In focus & Off focus \\
\hline Primary & $0.46(13.4 \%)$ & $1.01(21.3 \%)$ & & $0.65(25.4 \%)$ & $1.46(38.5 \%)$ \\
Scatter & $2.98(86.6 \%)$ & $3.73(78.7 \%)$ & & $1.91(74.6 \%)$ & $2.33(61.5 \%)$ \\
Total & $3.44(100 \%)$ & $4.74(100 \%)$ & & $2.56(100 \%)$ & $3.79(100 \%)$ \\
\hline \hline
\end{tabular}




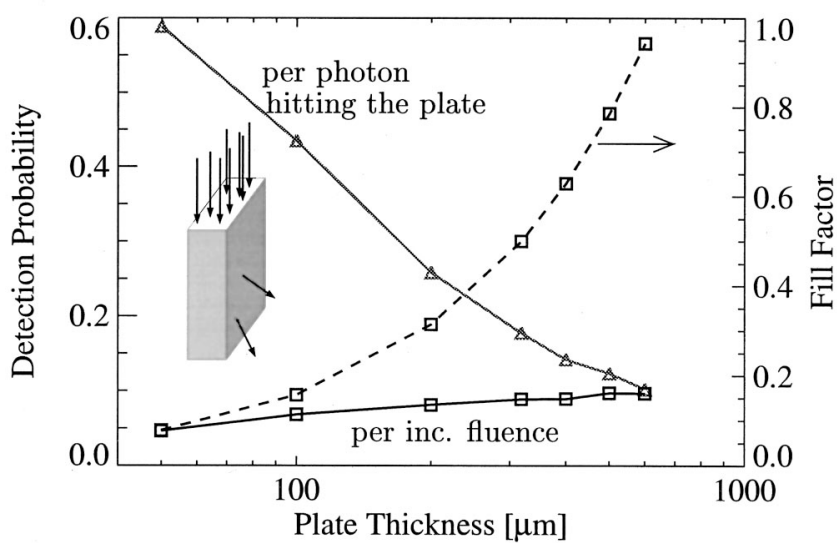

FIG. 6. Detection probability per photon hitting the plate (gray solid) and per incident fluence (black solid) as a function of plate thickness. The photons are hitting the plate perpendicularly to the surface. The fill factor (dashed line, right axis) is the ratio between the tungsten surface and the total surface exposed to the incident photons. The detection probability per incident photon fluence is then the product of the detection probability per photon hitting the plate and the fill factor.

roughly that on average every other photon produces at least one charged particle liberated from the plate and therefore producing charge in the xenon cavities.

It is interesting to note, that $p_{\text {det }}$ per incident photon fluence (assuming that the photons do not interact in xenon) is decreasing with decreasing plate thickness from about $10 \%$ for the $600 \mu \mathrm{m}$ plate to about $5 \%$ for the smallest $(50 \mu \mathrm{m})$ plate. This means that with decreasing plate thickness, the decreasing cross section outweighs the increasing number of liberated charged particles.

The situation described so far corresponds to an isolated tungsten plate in the center of the detector, where the beam hits the plate along its longitudinal axis. In the following, the efficiency parameters are studied for the entire arrangement of plates and cavities in the arc detector. Figures 7 and 8 show typical AED distributions for the arc detector with a

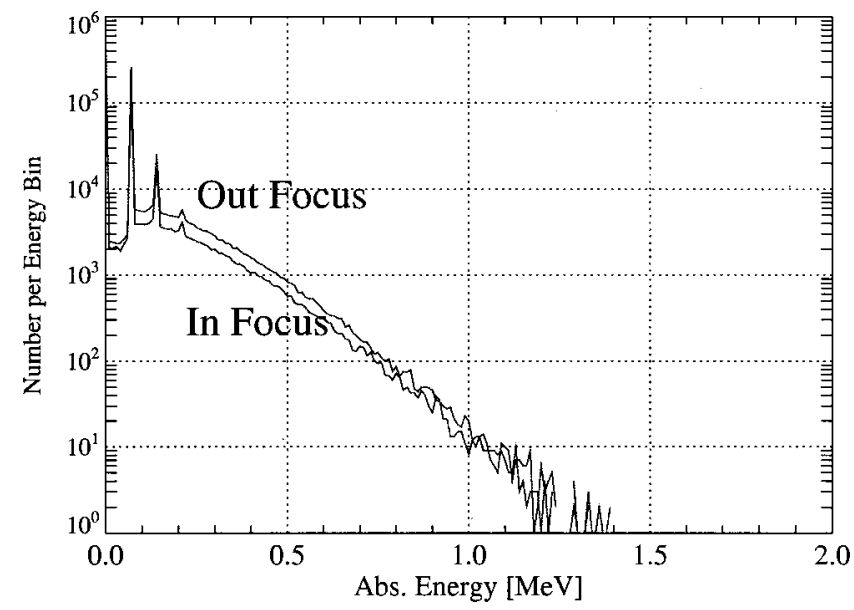

FIG. 7. Comparison of the absorbed energy distributions $N$. AED for the detector in and out of focus. The number of incident photon histories was $10^{6}$. The distributions include the noninteracting photons in the first energy bin.

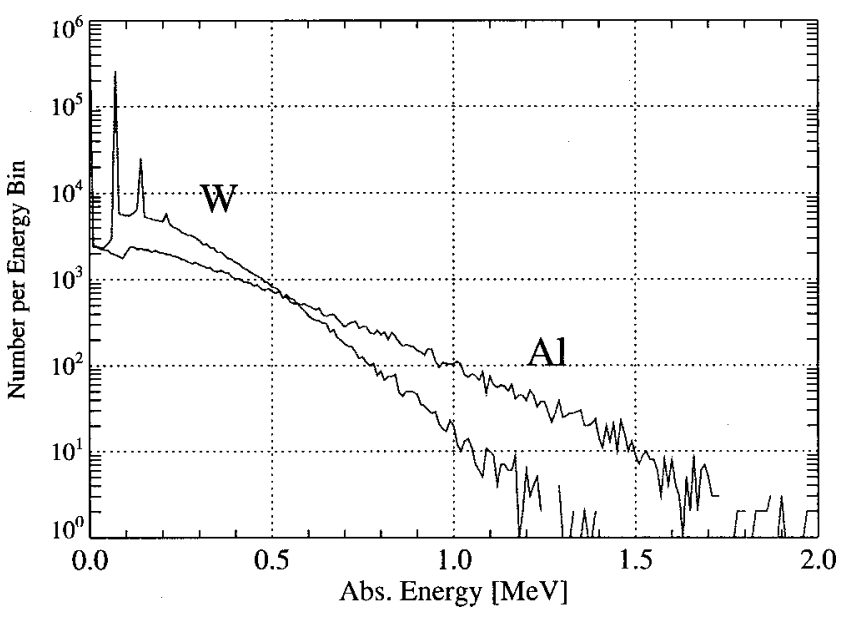

FIG. 8. Comparison of the absorbed energy distributions $N$. AED for a hypothetical detector with aluminum septa and for the detector with tungsten septa. The detector is placed out of focus with the photon source. The number of incident photon histories was $10^{6}$. The distributions include the noninteracting photons in the first energy bin.

plate thickness of $320 \mu \mathrm{m}$. The incident field size was 3.89 $\times 30 \mathrm{~cm}^{2}$ and the number of incident photons was $10^{6}$. From these distributions and with Eq. (3) the detective quantum efficiency at zero frequency $\mathrm{DQE}(0)$ and the quantum efficiency QE were computed, which are both given in Table II. The efficiency numbers given in the table are compiled as a function of the plate thickness (but fixed cell size of 1.28 $\mathrm{mm})$. In addition, for the in-focus position, a so-called array quantum gain factor was calculated. The quantum gain factor is the ratio between the calculated $\mathrm{QE}$ and $p_{\text {det }}$. It therefore indicates the signal amplifying effect of the arrangement of the plates in a linear array and is the multiplicative increase in probability that a single photon is detected in the detector structure rather than from a single plate.

In general, the efficiencies are higher for the detector placed out of focus. The main reason for this is that, as in the discussion above, the geometrical cross section for hitting a tungsten plate is increased with respect to the in-focus position. The efficiencies increase with increasing plate thickness for both detector positions. In addition, the array quantum gain factor increases, which indicates, that with a larger interaction volume (tungsten), a photon has an increased chance of liberating charged particles during its particle history. This effect, which was already apparent for the single plate, is even more pronounced in the detector array structure. Therefore, the quantum efficiency of the detector is increasing faster with increasing plate thickness than the detection probability per incident photon fluence of the single plate (Fig. 6).

From the calculated AEDs in Fig. 8 it can also be seen, that a hypothetical detector with aluminum septa plates should show a lower $\mathrm{DQE}(0)$ than the detector with the tungsten plates, as the energy distribution shows a wider spread, and therefore a larger second moment of the AED.

As an additional quality test of the results, QE and DQE(0) were calculated for various cutoff energies ECUT and PCUT. It was shown, that the efficiency figures were 
TABLE II. Efficiency numbers for the arc-shaped xenon gas detector for different plate thickness. QE is the quantum efficiency of the detector. For the in-focus position, $p_{\text {det }}$ is the detection probability in a single plate from Fig. 6. AQGF is the array quantum gain factor, which is the ratio between $\mathrm{QE}$ and $p_{\text {det }}$.

\begin{tabular}{|c|c|c|c|c|c|c|}
\hline \multirow[b]{2}{*}{ Plate width $[\mu \mathrm{m}]$} & \multicolumn{4}{|c|}{ In focus } & \multicolumn{2}{|c|}{ Out of focus } \\
\hline & $\mathrm{QE}[\%]$ & $p_{\text {det }}[\%]$ & AQGF & $\mathrm{DQE}(0)[\%]$ & $\mathrm{QE}[\%]$ & $\mathrm{DQE}(0)[\%]$ \\
\hline 50 & 8.1 & 4.6 & 1.8 & 5.9 & 16.5 & 12.2 \\
\hline 100 & 12.6 & 6.8 & 1.9 & 9.0 & 25.0 & 18.0 \\
\hline 200 & 20.6 & 8.1 & 2.5 & 14.5 & 36.2 & 25.6 \\
\hline 320 & 29.2 & 8.9 & 3.3 & 20.4 & 44.4 & 31.4 \\
\hline 400 & 34.7 & 8.9 & 3.9 & 24.4 & 48.1 & 34.1 \\
\hline 500 & 40.9 & 9.7 & 4.2 & 29.1 & 50.7 & 36.1 \\
\hline
\end{tabular}

unaffected by a change in ECUT and PCUT up to $611 \mathrm{keV}$ and $100 \mathrm{keV}$, respectively.

Figure 9 shows the total absorbed energy per source particle in all the xenon cavities for an incident thin slit beam as a function of plate thickness at two different locations on the detector (center and off-center). This number is a measure of the signal creation efficiency and represents a relative measure for the expected signal-to-noise ratio. The total signal creation is essentially proportional to the total path length of charged particles traversing the gas cavities. According to the chosen setup for the calculations, where the spacing between the plates was kept constant, the volume of the gas cavities decreases with increasing plate thickness. As was seen in Figs. 4 and 5, due to the increased number and energy of photons incident on the off-center tungsten plates, the number of produced charged particles is also higher, therefore resulting in an increased total absorbed energy. In the offcenter case, where the average path length of the photons through tungsten is much shorter than in the center, the vast majority of scattered, therefore lower-energetic, photons will also have an increased probability to interact and produce electrons, which leave the plates. A second effect, which contributes to the increasing absorbed energy with decreasing plate thickness is the increase in the signal-collecting xenon volume. As a consequence of these effects, the total absorbed energy is sharply increasing with decreasing plate thickness.

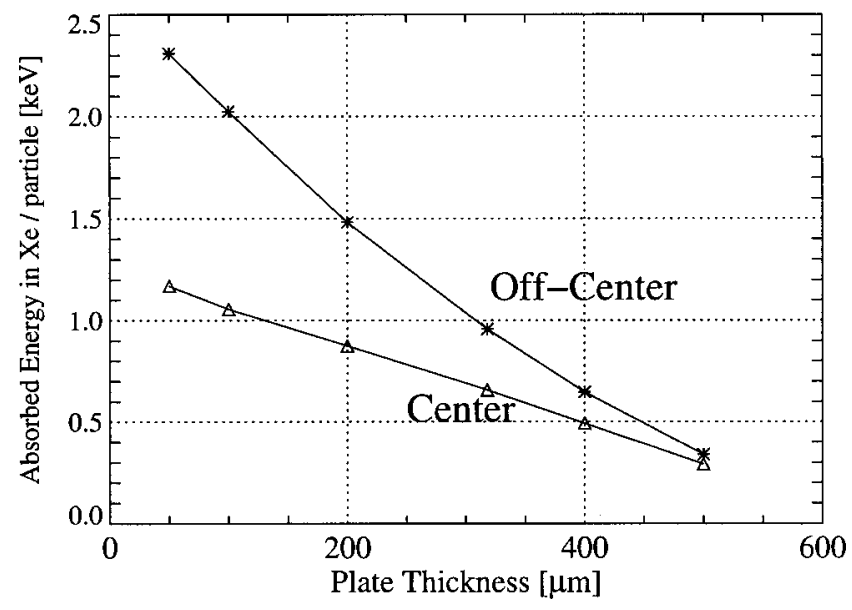

FIG. 9. Total absorbed energies in the xenon cavities from small beams covering one detector cell in the center and off-center.
In the center, this effect is less pronounced, as the increasing number of produced charged particles with increasing plate thickness is almost completely compensated by the decreasing path length through the cavities. Therefore, the slope of the line is purely given by the change in the xenon volume.

\section{Resolution}

Figure 10 shows the comparison between the measured and calculated responses for an incident finite-sized slit beam (small beam response). The incident photons are centered towards a collecting electrode. As the signal produced by the electrons liberated from the collecting electrode is collected by the same electrode, the measured and calculated line spread functions show a distinct maximum in a single detector channel. This would not be the case, if the center of the slit was centered towards a charged electrode, which would cause the maximum of the signal to be distributed over two channels.

It is seen from Fig. 10, that the measured and calculated responses agreed well within about 10 channels around the maximum. At larger distances from the maximum, the measured signal is slightly larger than the calculated signal. One explanation for this is, that the actual energy spectrum could have been slightly harder than in the calculations. This would

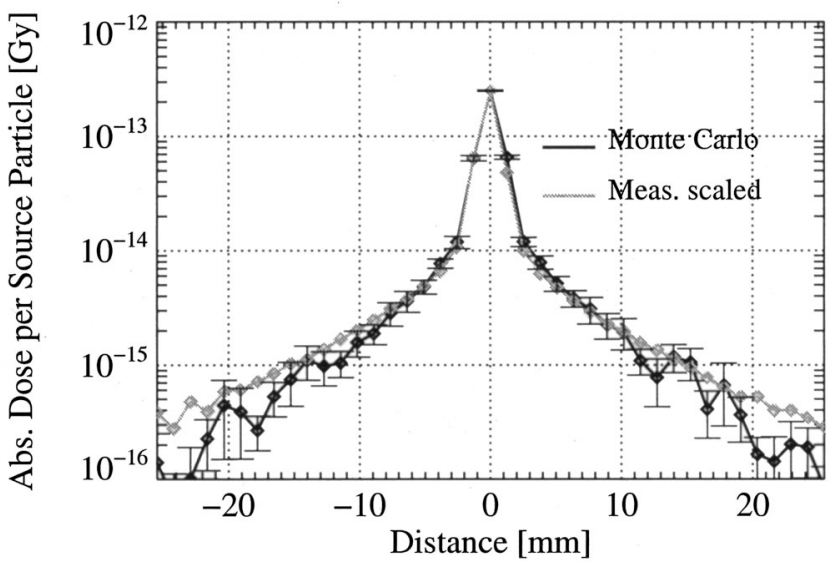

FIG. 10. Comparison of measured and calculated small beam responses. The slit beam is directed towards a collecting electrode. The measured signal is scaled to match the calculated value at the maximum. The error bars indicate the statistical error of the Monte Carlo calculation. 


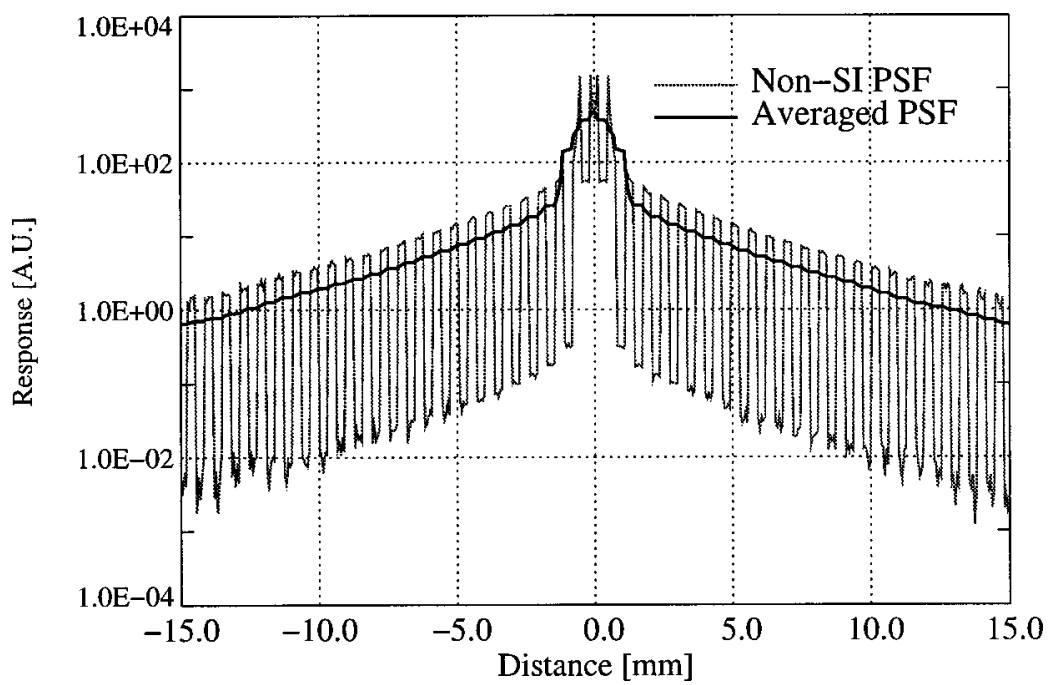

FIG. 11. Non-shift-invariant (Non-SI) and averaged presampled point spread functions in the center of the arc detector with $320 \mu \mathrm{m}$ thick tungsten plates. The tungsten plates are pointing towards the photon source. The averaged PSF is the non-shift-invariant PSF convolved with a box function of the size of one cell size.

result in increased side scatter. The differences are also influenced by the amount of background subtracted. However, the quality of the calculated small beam response profile was regarded to be sufficient for the calculation of the resolution parameters.

The non shift-invariant point response of the detector obtained with the described backward approach is illustrated in Fig. 11. It is seen, that steep maxima and minima occur depending on what material the pencil beam is incident on. A pencil beam hitting a tungsten plate creates a maximum, a pencil beam hitting a xenon cavity a minimum. The maxima and minima are a direct expression of the non-shiftinvariance. In the center, it is seen, that the signal being produced is higher, the closer the pencil is hitting the plate's lateral surface.

For the calculation of the presampled modulation transfer function, the non-shift-invariant response function cannot be used, as it would contain frequencies from the non-shiftinvariant input modulation. In order to circumvent this problem, a shift-invariant "averaged" point response function was constructed by convolving the non-shift-invariant response function by a box function of the width of one detector cell. The result is a spread function for an incident finitesized pencil beam covering one elementary symmetry cell of two xenon cavities and two tungsten plates and is therefore shift-invariant. This function is also shown in Fig. 11. The tails of the averaged PSF follow the tails of the maxima of the presampled non-shift-invariant PSF at approximately half their values. This is because half of all the pencils incident on a detector cell are incident on a xenon cavity.

The convolution operation with a box function in real space corresponds to a multiplication with a sinc-function in Fourier space. Therefore, the Fourier transform of the averaged PSF was divided by $\operatorname{sinc}(x / a)=a \sin (x / a) / x$, where $a$ is the width of the detector cell. The resulting modulation transfer functions are shown in Fig. 12. It is clearly seen, that the modulation transfer is in general higher for thicker plates. This is obvious from the fact, that thicker plates reduce side scatter in the detector.

\section{DISCUSSION AND CONCLUSIONS}

Triggered by the documented quality of reconstructed CT images from projections acquired with the arc-shaped xenon detector (Fig. 1 and Refs. 14 and 23), resolution and efficiency properties of this detector configuration were investigated. Especially dose response, quantum efficiency, detective quantum efficiency at zero frequency, line spread functions and modulation transfer functions were examined.

Concerning resolution, the decrease of the modulation transfer function with higher frequency is determined by the cell size of the detector and to a lesser extent by the thickness of the tungsten plates. Therefore, the modulation transfer is to first order independent of the location This shows, that a future version of the detector could benefit from a reduction in cell size.

It is easily seen, that the problem of the existence of a non-shift-invariant point response function should disappear if the angle between the incident $x$ rays and the orientation of the tungsten plates are larger than a certain "critical angle."

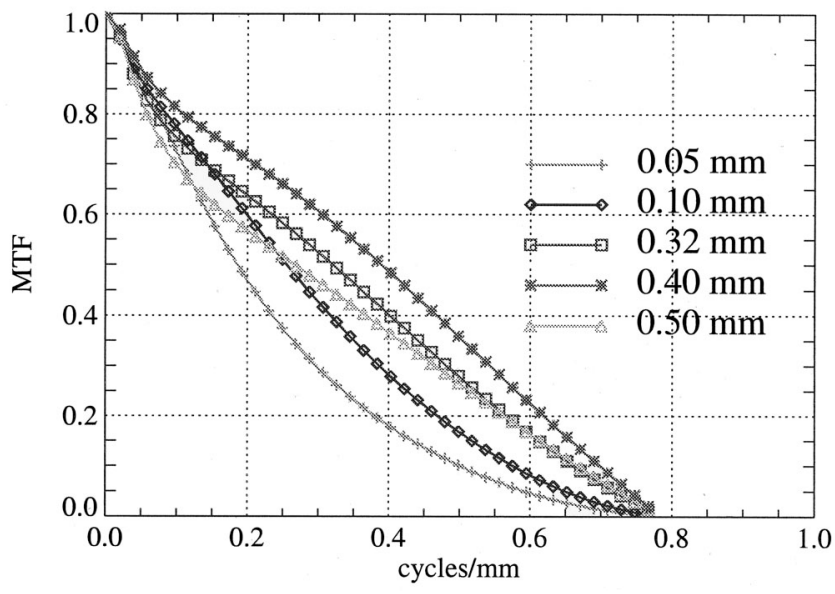

FIG. 12. Modulation transfer functions calculated from the averaged point spread functions of Fig. 11 and corrected by the sinc-function for plate thicknesses between 0.05 and $0.5 \mathrm{~mm}$ for a fixed cell size of $1.28 \mathrm{~mm}$. 
This angle can be defined as the angle, where an $\mathrm{x}$ ray just starts to intersect two tungsten plates (close to the region numbers 500 and 1000 in Fig. 4). Beyond this angle, the probability of interaction will be largely smoothed out over the region of the incident photons and it is expected, that the presampled point spread function becomes more continuous. The non-shift-invariant modulation transfer and its dependency on the detector location shall be discussed in greater detail in future work.

Concerning efficiency, the figures for $\mathrm{QE}$ and $\mathrm{DQE}(0)$ in Table II imply, that the combination of high- $Z$ materials together with a gas or another active medium leads to a very high efficiency for megavoltage radiation in the first two stages of the described detection system. A high DQE(0) results from a high quantum efficiency and the fact, that the charged particles lose their energy only in small amounts. This is a general observation for a gas as the active medium, as the probability of a catastrophic event (Möller or Bhaba scattering) within the small volumes of the gas cavities is low, therefore keeping the distribution of absorbed energies (and the second moment of the AED) small.

Due to the large field size used for the calculations, QE and $\mathrm{DQE}(0)$ in Table II are averaged quantities over the entire detector structure. For the detector placed in focus with the photon source, the numbers are independent of the field size and independent of the incident location on the detector. This is not the case for the detector placed out of focus. The efficiency parameters are dependent on the field size and whether a beam hits a center cell or an off-center cell. For a small beam incident on the center of the detector, the numbers are the same as for the detector placed in focus. The spatial dependency of the efficiency for the detector placed out of focus seems not to influence the quality of the reconstructed CT images, but further tests are required.

The optimal dimensions for the plate thickness and cell size have to be chosen recognizing the trade-off between resolution and efficiency. It appears from Table I that thicker plates would be preferable to thinner plates. However, as seen in Fig. 9, the total absorbed energy is decreasing with increasing plate thickness. The reason for this is the decreasing volume of the signal collecting medium (xenon gas cavities) with increasing plate thickness due to the constant spacing between the plates. In addition, thinner plates are associated with inferior resolution (cf. Fig. 12). However, from this figure, it seems, that there might exist an optimal plate thickness with respect to maximal resolution at around $0.4 \mathrm{~mm}$. The exact interplay between efficiency and resolution parameters for variable cell size and different septa plate materials will be addressed in greater detail in a later study.

For a given signal-to-noise ratio in the reconstructed image, the dose needed is inversely proportional to the quantum efficiency of the detector. ${ }^{24}$ The results in this paper indicate, that the high quantum efficiency of the arc chamber will lead to a significant reduction in dose compared to existing techniques for megavoltage radiation CT imaging. In addition, the off-focus arrangement of the detector with respect to the photon source causes the detector to be even more efficient than a detector positioned in focus with the source.
The detector in its current version is connected to a data acquisition system (DAS). The read-out electronics of the DAS is fast enough to acquire the data pulse by pulse up to $1000 \mathrm{~Hz}$. This together with the high efficiency and sufficient resolution is important for obtaining high-quality timeresolved information during the treatment, making the detector an ideal candidate for megavoltage CT imaging and image-guided radiotherapy.

\section{ACKNOWLEDGMENTS}

The authors thank Dave Pearson, Guang Fang, and Jeff Kapatoes from Tomotherapy, Inc. for insightful discussions. We would also like to thank the National Institute of Health (ROI CA 48902) and the Department of Energy (DOE DEFG07-ID13918).

a) Author to whom correspondence should be addressed. Electronic mail: keller@madrad.radiology.wisc.edu

${ }^{1}$ J. Leong and D. Shimm, "A method for consistent precision radiation therapy," Radiother. Oncol. 3, 89-92 (1985).

${ }^{2}$ D. G. Lewis, W. Swindell, E. J. Morton, P. M. Evans, and Z. R. Xiao, “A megavoltage CT scanner for radiotherapy verification,” Phys. Med. Biol. 37, 1985-1999 (1992).

${ }^{3}$ M. A. Mosleh-Shirazi, W. Swindell, and P. M. Evans, "Optimization of the scintillation detector in a combined 3D megavoltage CT scanner and portal imager," Med. Phys. 25, 1880-1890 (1998).

${ }^{4}$ M. Mosleh-Shirazi, P. Evans, W. Swindell, S. Webb, and M. Partridge, "A cone-beam megavoltage CT scanner for treatment verification in conformal radiotherapy," Radiother. Oncol. 48, 319-328 (1998).

${ }^{5} \mathrm{H}$. Guan and Y. Zhu, "Feasibility of megavoltage portal CT using an electronic portal imaging device (EPID) and a multi-level scheme algebraic reconstruction technique (MLS-ART)," Phys. Med. Biol. 43, 2925-2937 (1998)

${ }^{6}$ B. Hesse, L. Spies, and B. Groh, "Tomotherapeutic portal imaging for radiation treatment verification," Phys. Med. Biol. 43, 3607-3616 (1998).

${ }^{7}$ L. Spies, M. Ebert, B. Groh, B. M. Hesse, and T. Bortfeld, Quantitative cone beam megavoltage CT, in Proceedings of the 13th ICCR, The Use of Computers in Radiation Therapy, Heidelberg, Germany, May 2000, edited by W. Schlegel and T. Bortfeld (Springer, Berlin, 2000), pp. 150152 .

${ }^{8}$ S. Midgley, R. Millar, and J. Dudson, "A feasibility study for megavoltage cone beam CT using a commercial EPID," Phys. Med. Biol. 43, 155-169 (1998).

${ }^{9}$ B. A. Groh, J. H. Siewerdsen, D. G. Drake, J. W. Wong, and D. A. Jaffray, $\mathrm{MV}$ and $\mathrm{kV}$ cone-beam CT on a medical linear accelerator, in Proceedings of the 13th ICCR, The Use of Computers in Radiation Therapy, Heidelberg, Germany, May 2000, edited by W. Schlegel and T. Bortfeld (Springer, Berlin, 2000), pp. 561-563.

${ }^{10}$ T. R. Mackie, T. Holmes, S. Swerdloff, P. Reckwerdt, J. O. Deasy, J. Yang, B. Paliwal, and T. Kinsella, "Tomotherapy: a new concept for the delivery of dynamic conformal radiotherapy," Med. Phys. 20, 1709-1719 (1993).

${ }^{11}$ G. H. Olivera, D. M. Shepard, K. Ruchala, J. S. Aldridge, J. M. Kapatoes, E. E. Fitchard, P. J. Reckwerdt, G. Fang, J. Balog, J. Zachman, and T. R. Mackie, Tomotherapy, in Modern Technology of Radiation Oncology, edited by J. Van Dyk (Medical Physics Publishing, Madison, WI, 1999), pp. 521-587.

${ }^{12}$ K. J. Ruchala, Ph.D. thesis, University of Wisconsin, Madison, WI, 2000.

${ }^{13}$ D. A. Jaffray, D. G. Drake, M. Moreau, A. A. Martinez, and J. W. Wong, "A radiographic and tomographic imaging system integrated into a medical linear accelerator for localization of bone and soft-tissue targets," Int. J. Radiat. Oncol., Biol., Phys. 25, 773-789 (1999).

${ }^{14}$ K. J. Ruchala, G. H. Olivera, J. M. Kapatoes, E. A. Schloesser, P. J. Reckwerdt, and T. R. Mackie, "Megavoltage CT image reconstruction during tomotherapy treatments," Phys. Med. Biol. 45, 3545-3562 (2000). 
${ }^{15}$ A. L. Boyer, L. Antonuk, A. Fenster, M. Van Herk, H. Meertens, P. Munro, L. E. Reinstein, and J. Wong, "A review of electronic portal imaging devices (EPIDs)," Med. Phys. 19, 1-16 (1992).

${ }^{16}$ D. Yan, F. Vicini, J. Wong, and A. Martinez, "Adaptive radiation therapy," Phys. Med. Biol. 42, 123-132 (1997).

${ }^{17}$ D. A. Jaffray, D. Yan, and J. W. Wong, "Managing geometric uncertainty in conformal intensity-modulated radiation therapy," Semin Radiat. Oncol. 9, 4-19 (1999).

${ }^{18} \mathrm{~J}$. Balog, Ph.D. thesis, University of Wisconsin, Madison, WI, 1998.

${ }^{19}$ D. W. O. Rogers, B. A. Faddegon, G. X. Ding, C.-M. Ma, J. We, and T. R. Mackie, "BEAM: A Monte Carlo code to simulate radiotherapy treatment units," Med. Phys. 22, 503-524 (1995).

${ }^{20}$ I. A. Cunningham, M. S. Westmore, and A. Fenster, "A spatial-frequency dependent quantum accounting diagram and detective quantum efficiency model of signal and noise propagation in cascaded imaging systems," Med. Phys. 21, 417-427 (1994).

${ }^{21}$ D. A. Jaffray, J. J. Battista, A. Fenster, and P. Munro, "Monte Carlo studies of $\mathrm{x}$-ray energy absorption and quantum noise in megavoltage transmission radiography," Med. Phys. 22, 1077-1088 (1995).

${ }^{22}$ R. Swank, "Absorption and noise in x-ray phosphors," J. Appl. Phys. 44. 4199-4203 (1973)

${ }^{23}$ K. J. Ruchala, G. H. Olivera, J. M. Kapatoes, E. A. Schloesser, P. J. Reckwerdt, and T. R. Mackie, "Megavoltage CT imaging as a by-product of multileaf collimator leakage," Phys. Med. Biol. 45, N61-N70 (2000).

${ }^{24} \mathrm{H}$. H. Barrett and W. Swindell, Radiological Imaging-The Theory of Image Formation, Detection and Processing (Academic, New York, 1981), Vol. 1. 